\title{
The role of direct parameter specification and attentional capture in near-threshold priming of motor reactions
}

\author{
Blandyna Skalska ${ }^{1}$, Piotr Jaśkowski ${ }^{1}$, and Rob H. J. van der Lubbe ${ }^{2}$ \\ ${ }^{1}$ Department of Cognitive Psychology, University of Finance and Management Warszawa, Poland \\ 2 Department of Cognitive Psychology, University of Twente, Enschede, The Netherlands
}

Received 05.05.2005

Accepted 05.01.2006

\section{Keywords}

Subliminal priming, direct parameter specification, visuo-spatial attention

\section{ABSTRACT}

The priming of motor responses can be induced by preceding visual stimuli that have been made invisible by metacontrast masking ('primes'). According to the concept of direct parameter specification (DPS; Neumann, 1990), strong similarity between prime and target results in the processing operations that are to be applied to the target being also induced by the prime. As targets have to be attended to, this also implies that attention is captured by the location of a prime, thereby facilitating motor priming effects. This hypothetical effect may be viewed as a form of top-down attentional capture. In some subliminal priming experiments (e.g. Jaśkowski, Skals- ka, \& Verleger, 2003), however, attentional capture may have been unrelated to target identity, as stimuli with unique features (singletons) are known to induce bottom-up attentional capture. Three experiments were performed that largely confirmed the view that the results of these earlier experiments were due to top-down attentional capture, in line with DPS. However, the priming effect was also evoked by a singleton irrelevant to the participants' task, although this effect was weaker than in case of strong similarity between prime and target. Priming effects remained when singletons were absent from one side of the visual field, suggesting that the presence of singletons is not a requirement for the observation of motor priming effects.

\section{INTRODUCTION}

\section{Motor reaction priming by masked stimuli}

Numerous studies have shown that responses to stimuli that are preceded by subliminal (i.e., not consciously reportable) stimuli are affected by specific features of the subliminal stimuli. In the classical experiments by Neumann and Klotz (1994) a target display was used consisting of two figures, a square and a diamond, in which either the square or the diamond appeared to the left or right of fixation. One of the figures was defined as the target, which required a left or right key press depending on its side relative to fixation. The other nontarget figure was a distractor. The target display was preceded by a prime display, which consisted of two shapes which were small replicas of the diamond and square. This prime display was made unrecognizable by metacontrast masking induced by the target display. Results showed that responses were speeded up when the side of the target-like replica in the prime display corresponded with the side of the target in

Corresponding author: Blandyna Skalska, Department of Cognitive Psychology, University of Finance and Management ul. Pawia 55, 01-030 Warszawa, Poland, tel. +48-880013971, blandynaskalska@vizja.pl 
target display (compatible primes) as compared to when they did not correspond (incompatible primes). This finding was replicated in several studies (Ansorge \& Neumann, 2005; Jaśkowski et al., 2003; Jaśkowski, van der Lubbe, Schlotterbeck, \& Verleger, 2002; Klotz \& Neumann, 1999; Klotz \& Wolff, 1995).

Neumann and Klotz (1994) proposed that subliminal primes invoked the preparation of the motor action specified by their form, that is compatible primes activated the required motor action whereas incompatible primes activated an erroneous motor action. This notion seems to be supported by EEG data. Specifically, motor activation evoked by masked, unidentifiable primes has been observed in the lateralized readiness potential, with target-like replicas in prime displays evoking activity corresponding to the associated response in the contralateral motor cortex (Jaśkowski et al., 2002; Leuthold \& Kopp, 1998). Thus, it may be proposed that the response delay on incompatible trials is caused by the suppression of inadequate motor actions triggered by the prime. We will first focus on this hypothesis.

\section{Direct parameter specification}

The direct parameter specification (DPS) hypothesis originally proposed by Neumann (1990) was invoked by Neumann and Klotz (1994) to explain the subliminal priming of motor actions. According to DPS, "input information specifies action parameters without (or at least before) giving rise to a corresponding mental representation as a necessary prerequisite" (Neumann, 1990; p. 212). In a more recent paper (Neumann, Ansorge, \& Klotz, 1998), it is stated that once an action plan is completed, sensory information can be used to specify free parameters of the responses without mediating conscious perception. Nevertheless, the specified parameters may sometimes need to be corrected off-line when something goes wrong (Jaśkowski et al., 2003). An important consideration is the idea that DPS only occurs when the prime resembles the target. Accordingly, Ansorge, Heumann, and Scharlau (2002) claimed that the priming effect diminished when figures in the prime were made dissimilar to the target. Hence, response preparation evoked by subliminal primes requires feature overlap between the prime and the target display.

Interestingly, recent work has related DPS to attentional orienting, proposing that DPS may be involved in the control of attention and choice responses alike. Specifically, it has been argued that attention is unconsciously captured by the prime when it matches features of the target (e.g., see Scharlau \& Ansorge, 2003). In some specific settings, however, DPS may only be a part of the explanation. For example, in a few subliminal priming experiments (e.g. Ansorge \& Neumann, 2005; Jaśkowski et al., 2003) primes may have exerted their effects because the target-like replica in the prime automatically attracted attention due to its high salience. These two different possibilities will be fleshed out in the following section.

\section{The possible influence of attentional capture}

Attention is known to be effectively captured by the abrupt onset of a stimulus. For example, in a study by Posner and Cohen (1984), to-be-detected targets, presented left or right from fixation, were preceded by uninformative peripheral cues to the left or right. Reaction times (RTs) were shorter when the target occurred on the side of the peripheral cue than when it occurred on the other, uncued side; a result that is ascribable to attentional capture by the peripheral cue. Miller (1989) showed that this effect also occurs when abrupt offsets are used, suggesting that attention can be captured by any abrupt changes such as onsets or offsets (cf. Franconeri, Simons, \& Junge, 2004). Theeuwes (1992; 2004) additionally provided evidence that stimuli with unique and salient features (e.g., a red item among blue ones) or so-called singletons can automatically capture attention. This form of attentional capture, guided by discontinuities in the environment, has been labeled bottom-up or exogenous attentional orienting, to signify that this attentional capture is automatic and not dependent on intentions. Indeed, in some studies it was shown that attention may be captured by abrupt onsets in the environment even when the relevant target location is already indicated by a preceding, $100 \%$ valid cue (e.g., van der Lubbe \& Postma, 2005, but see Theeuwes, 1991) ${ }^{1}$.

Importantly, in some experiments, exogenous orienting may provide an alternative explanation for the priming effect, without the requirement of DPS. In the following this view will be denoted as bottomup attentional capture. We will focus on an example from Jaśkowski et al.'s experiments (2003). In their experiments, stimuli consisted of two square outlines placed to the left and right of fixation (see Fig. 1). The intact outline was defined as the distractor, and the outline with 3-mm gaps to the left and right was defined as the target. The target display was preceded by a sequence of four stimuli: one prime display in which the target-like replica was displayed on the same side as, or the opposite side to, the target and 
three filler displays (pairs of intact outlines). Thus, the target-like replica was embedded in a stream of homogeneous figures. Due to this homogeneity the target-like replica may have acted as a singleton, thereby automatically attracting attention, which may have invoked a larger priming effect. Indeed, in Jaśkowski et al.'s study, the priming effect was larger than in other experimental settings ( $100 \mathrm{~ms}$ vs. 10 to $70 \mathrm{~ms}$ in other studies: Kunde, 2003; Leuthold \& Kopp, 1998; Lingnau \& Vorberg, 2005; Mattler, 2003; Neumann \& Klotz, 1994; Schlaghecken \& Eimer, 2002), which may be due to the contribution of bottom-up attentional capture.

Alternatively, attentional capture may also be understood from the perspective of DPS, as attention may be captured by the prime when it contains features that match features of the target (e.g., see Scharlau \& Ansorge, 2003). This view will be denoted as top-down attentional capture. Highly relevant for this conception is the top-down view of attentional capture forwarded by Folk, Leber, and Egeth (2002). They stated: "there is growing evidence that the efficient allocation of visual selective attention involves a delicate interplay between the properties of the stimulus itself and the behavioral goals of the observer". In their seminal study, Folk, Remington, and Johnston (1992) showed that irrelevant abrupt onsets only captured attention when the target also had an abrupt onset. When the target was defined by a unique color, abrupt onsets no longer affected performance. By the same token, an abrupt change of the color of a target letter in a visual search task did not improve performance (Gibson \& Jiang, 1998), and a cue was more effective in capturing attention when its color matched one of the possible target colors (Ansorge \& Heumann, 2003). Thus, combining DPS with the view on attentional capture by Folk et al. $(2002 ; 1992)$ leads to the following proposal: Attention is attracted to the location of the target-like replica in the prime display due to featural overlap with the target, which facilitates the processing of the subsequent target when it occurs on the same side. Moreover, according to Folk et al. (2002; 1992), attentional capture is always contingent on target features, which implies that pure bottom-up effects do not really exist.

Although attentional capture may be involved, either in a pure bottom-up manner or in a more top-down manner, it may be questioned whether subliminal stimuli can actually capture attention. McCormick (1997), however, provided an affirmative answer to this question. He showed that invisible peripheral cues still had a significant effect on RT to targets displays (rendering RTs longer or shorter depending on cue validity).
In the present experiments, we examined whether bottom-up attentional capture (induced by discontinuities) can account for the priming effects in specific types of subliminal priming experime nts. The bottom-up account may apply in experiments that employ peripherally presented stimuli in which the target contains a unique salient feature that is not contained in the distractor. The bottom-up account would claim that priming effects are solely due to the salience of the target-like replica in the prime display. Conversely, according to the top-down attentional capture hypothesis, attention should be attracted only by a target-like replica in the prime display because of feature overlap with the target ${ }^{2}$.

\section{GENERAL METHOD}

\section{Participants}

Participants were recruited from the population of psychology students of Kazimierz Wielki University in Bydgoszcz. They obtained course credit for their participation. All had normal or corrected-to-normal visual acuity and no known visual dysfunctions.

\section{Apparatus}

All stimuli were presented on a 17-in. monitor $(85 \mathrm{~Hz}$ in Experiment 1 and $150 \mathrm{~Hz}$ in Experiments 2 and 3) driven by Presentation software (Neurobehavioral Systems v. 0.55). The observation distance was $75 \mathrm{~cm}$. Participants responded with two keys of the computer keyboard (left Ctrl and numerical Enter).

\section{Stimuli and procedure}

The stimuli used in the experiments were similar to those used by Jaśkowski et al. (2003). Three kinds of squares, either with intact outlines, or with $3-\mathrm{mm}$ gaps to the left and right or 3-mm gaps at the top and bottom, were employed. Displays consisted of two squares placed to the left and to the right of fixation. In every trial, five displays were presented one after another. The square in each successive display was a slightly larger copy of the previous one (although the gaps were identical in all displays), thereby invoking metacontrast masking. Squares of the first display subtended $1.2 \times 1.2^{\circ}$, increasing to $2.9 \times 2.9^{\circ}$ in the final display (Fig. 1). The center of each square was placed $1.6^{\circ}$ to the left or to the right of fixation. The first four displays were each presented for $7 \mathrm{~ms}$ (12 ms in Exp. 1). The onset asynchrony between each 
of these displays was $33 \mathrm{~ms}$ (35 ms in Exp. 1), which meant that the displays appeared 133, 100, 67, and $33 \mathrm{~ms}$ before the target display $(140,105,70$, and $35 \mathrm{~ms}$ in Exp. 1). In each experiment, one stimulus (either the intact square or the square with gaps) was defined as the target. In the neutral condition, there were four filler displays consisting of pairs of intact squares. The experiments were performed in a soundproof and dark room. For other details see the Method section of each experiment and the Figures.

Each experiment consisted of two parts. In the first RT part, participants were required to make speeded reactions to the target display with the index finger on the button at the target side, with reaction times and error rate as dependent measures. The stimulus defined as the target occurred with equal probability at the left or the right side. Participants had to respond as fast and accurately as possible. In the second part, participants were required to detect or identify a figure in the prime display. This task we denoted as "prime identification task" (PI) with "percent correct identification" (PCI) as the dependent measure. Participants were informed about the precise structure of the prime stimuli. The stimuli were identical to the RT part. Feedback about the correctness of responses was provided only in Experiment $1^{3}$.

\section{Statistical analysis}

\section{Identification}

As the identification ability of individual participants differed markedly, we decided to report data for all participants irrespective of their performance in the PI task and to show separately the data for participants who performed at chance level in the PI task (see Appendix). Importantly, the pattern of results was independent of whether the analyses included data of all or only the selected participants. Correlations between the priming effect and PCI were nonsignificant in Experiment 1 and 2, and significant only in Experiment 3. The neutral condition in which no singleton was flashed in a prime display was provided only for illustrative purposes and was not included in the statistical analysis.

\section{Reaction times and error rates}

Mean RTs from correct responses and error rates were statistically evaluated with repeated measures analyses of variance (ANOVAs). When appropriate, Huynh-Feldt adjustments to the degrees of freedom were performed. We report whether $p$ values were below .05, or even lower than .001 .

\section{EXPERIMENT 1}

Our first experiment aimed to test whether a subliminal priming effect can be successfully evoked by a target-like replica in the prime display when it contains no salient features. Although it was not the case in the original procedure by Neumann and Klotz, in some experiments, the prime is not only similar to the target but also possesses a salient feature (Ansorge \& Neumann, 2005; Jaśkowski et al., 2003; Miśkiewicz, Skalska, \& Jaśkowski, 2002; Skalska, Gierszewska, Miłkowska, Okulicz, \& Jaśkowski, 2004). As a consequence, in those studies attention may have been attracted towards the target-like replica location either due to its similarity with the target (top-down attentional capture) or because it possessed a salient feature (bottom-up attentional capture). This coupling was broken down in Experiment 1. Similar stimulation conditions were used in the first experiment of Jaśkowski et al.'s study (2003).

In our experiment, we changed the instruction by asking participants to react to the side of the intact square rather than to the square with gaps. This required the target-like replica to be displayed seven times in the priming sequence and the salient distractor (a square with gaps on its vertical sides) only once (we refer to it as the prime display). If bottom-up attentional capture plays a key role in the priming effect, then reactions should be facilitated when the distractor occurs on the side of the target (i.e., with incompatible target displays). According to the topdown attentional capture account, however, reactions should be facilitated when the distractor occurs on the non-target side (i.e., with compatible target displays).

\section{Method}

\section{Participants}

Eighteen students took part in the experiment.

\section{Stimuli and procedure}

The intact square was defined as the target, and the square with gaps was defined as the distractor. Thus, each target display was preceded by three target-like replica pairs (filler displays) and one prime pair consisting of a target-like replica and a distractor (Fig. 1). A trial was defined as compatible when the target in the target display and the target-like replica in the prime display were on the same side. This implies that the same sequence of stimuli in the current study and in Experiment 1 of Jaśkowski et al. (2003) 


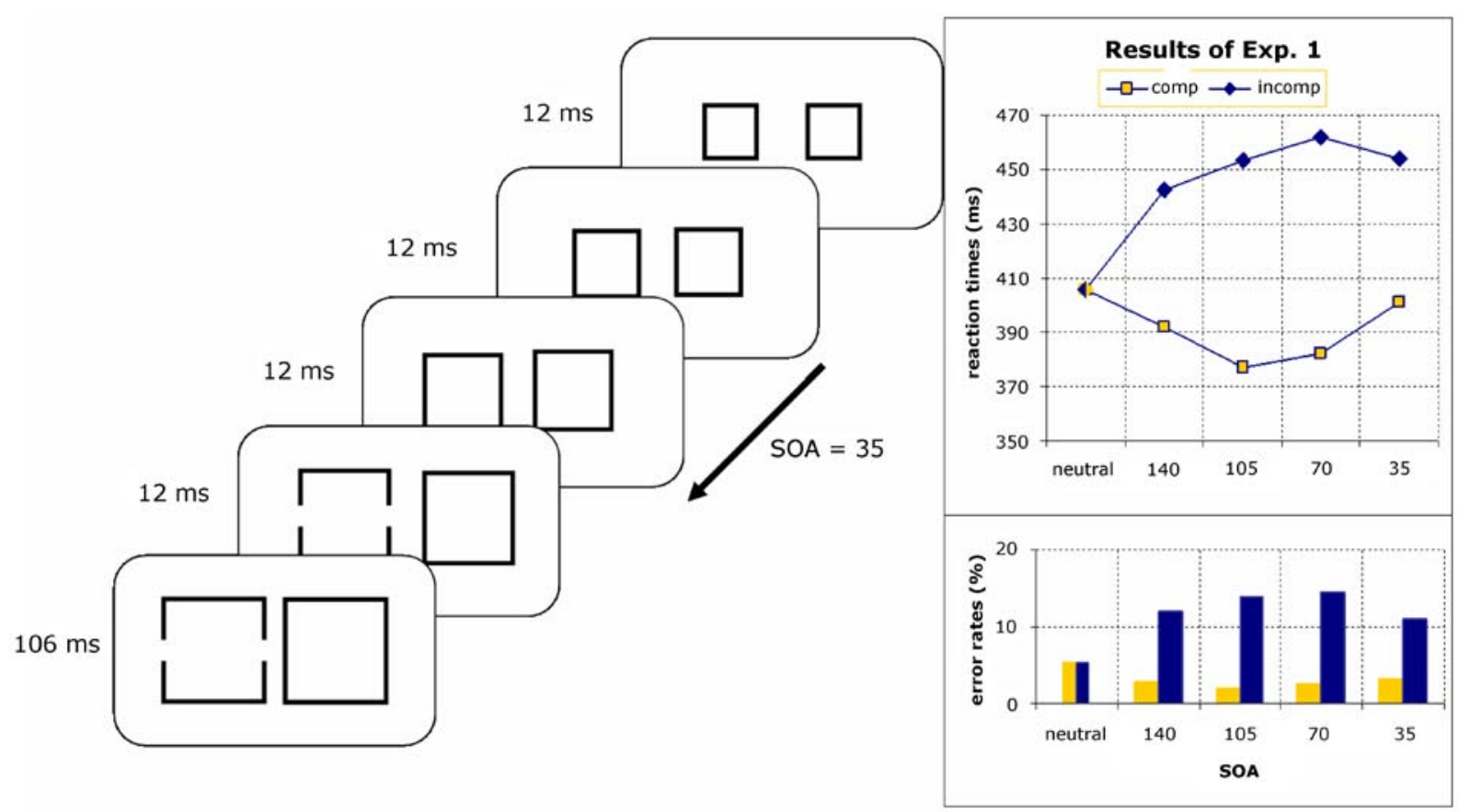

\section{Figure 1.}

Left: The sequence of stimulus displays used in Experiment 1. An example of a compatible trial is shown in which a right button press was required. Right: Reaction times and error rates in Experiment 1 as a function of compatibility and temporal distance (SOA) between a prime display and the target display.

are considered as compatible or incompatible, but that they differ with regard to the side of the target. Each display was presented for $12 \mathrm{~ms}$, but the target display was presented for $106 \mathrm{~ms}$. The critical prime was displayed with one of four possible stimulus onset asynchronies (SOAs; 140, 105, 70, and $35 \mathrm{~ms}$ ). For each of nine possible conditions (compatibility $x$ SOA plus one neutral condition) 40 trials were presented.

In each trial of the prime identification part, two sequences of stimuli were presented: one with and one without the distractor in the priming sequence. Participants had to indicate which one contained the distractor. In total, 160 trials were presented.

\section{Results}

\section{Identification}

Eight out of 18 persons chose the stimuli that contained the distractor better than may be expected on the basis of chance. However, the correlation between the priming effect and PCI was not statistically significant for any of the four SOA conditions; $r$ values ranged from -0.4 to 0.1 and ps from 0.09 to 0.9. Possibly, this higher PCI is partially caused by learning during the prime identification test. Being well motivated, our participants may have used the correctness feedback to improve their effectiveness at guessing (see also Schubö, Schlaghecken, \& Meinecke, 2001; Sobieralska \& Jaśkowski, 2005). Post-experimental debriefing revealed that this task was very difficult for participants. Many of them also admitted that they did not notice the distractor in the priming sequence in the first part (RT part). All data were included in the following reported analyses. For the analyses reported in the Appendix, we included only the data of those participants who detected the distractor at a level that was not significantly different from chance.

\section{Reaction times}

Mean RTs were shorter for compatible than for incompatible trials (388 vs. $453 \mathrm{~ms}$ ), $F(1,17)=210.2$, $p<.001$. RT for neutral trials was $412 \mathrm{~ms}$. RTs de-pended on SOA, being longer for shorter SOAs, $F(3,51)=5.8, p<.05$. The priming effect depended also on SOA, being largest for medium SOAs, that is, 105 and $70 \mathrm{~ms}$ [RT(incompatible) - RT(compatible) $=76$ and $80 \mathrm{~ms}$, respectively]; Compatibility $\times$ SOA interaction, $F(3,51)=10.2, p<.001$ (Fig. 1).

Error rates (Fig. 1) were higher in incompatible than in compatible trials $(11$ vs. $3 \%), F(1,17)=26.6 p<.001$. The error rate for neutral trials was $5 \%$. No other reliable main effect or interaction was found. 


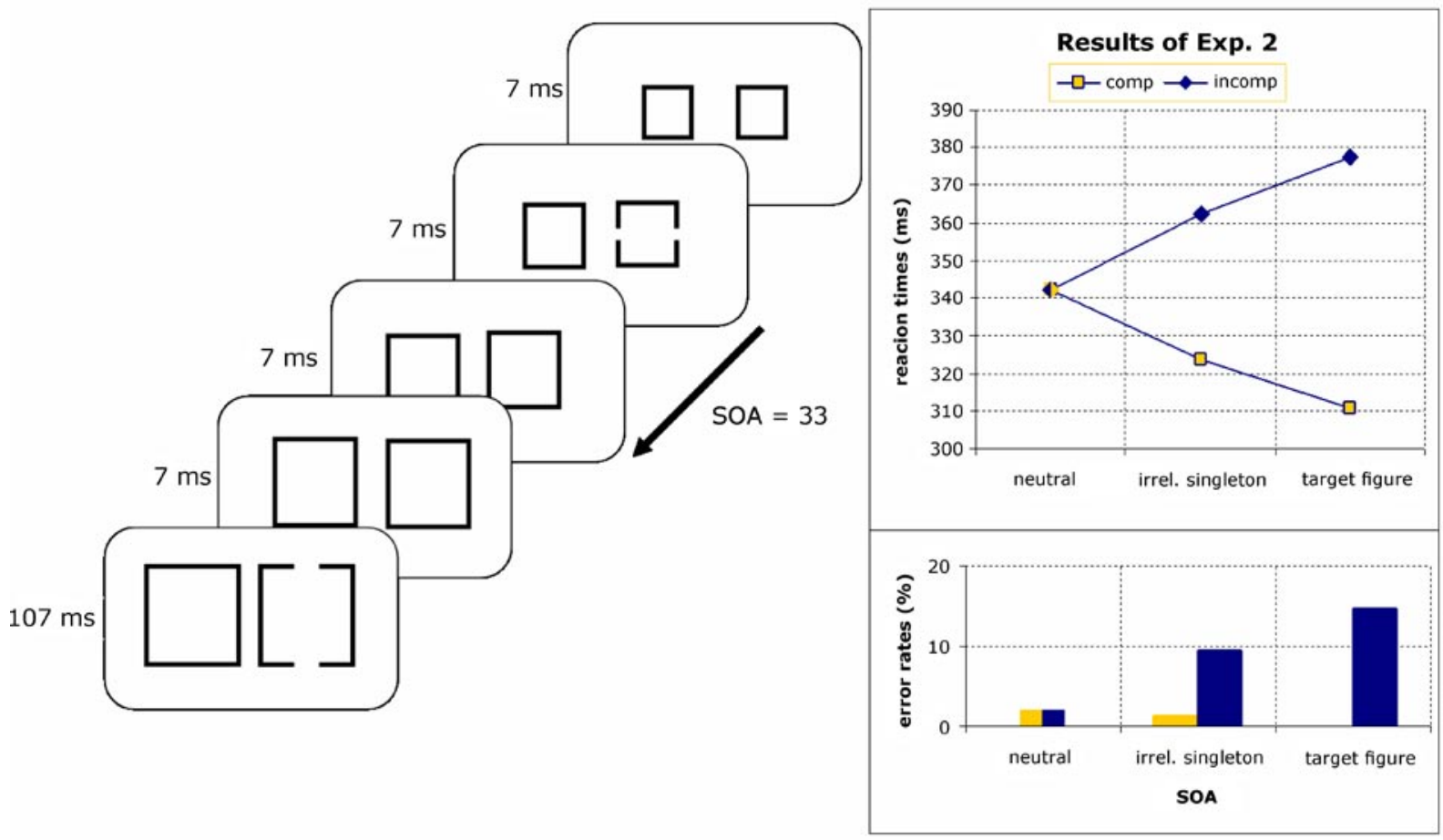

\section{Figure 2.}

Left: The sequence of stimuli used in Experiment 2. An example of a compatible trial is shown in which a right button press was required. The prime display contains an irrelevant singleton whose position corresponds with the side of the target in the target display. Right: Reaction times and error rates in Experiment 2 as a function of compatibility and type of singleton (target-like vs. irrelevant).

\section{Discussion}

In Experiment 1, we tested whether bottom-up attentional capture by a salient distractor could provide a better account of the observed priming effects than top-down attentional capture by opposing their hypothetical effects. The results (RTs and error rates) are well in line with the view of top-down attentional capture, as responses were faster and more accurate for compatible than for incompatible prime displays. Despite these clear effects, we cannot fully exclude the possibility that bottom-up attentional capture was involved, as it might modulate priming effects to some extent. In other words, the priming effect may be weaker when the target contains no unique features. To further explore the possible contribution of bottomup attentional capture, we performed two additional experiments.

\section{EXPERIMENT 2}

In Experiment 2, the target display was preceded by three neutral displays with distractors (filler displays), and one prime display consisting either of a target-like singleton and a distractor, or of a ro- tated version of the target-like singleton that had no overlap with the target (an irrelevant singleton) and a distractor. Both singletons were considered to be comparable in saliency, which implies that their capacity to induce bottom-up attentional capture is the same.

According to the top-down attentional capture account, the irrelevant singleton should have little effect upon behavior, as feature overlap with the target is minimal. Conversely, according to the bottom-up attentional capture account, the irrelevant singleton should affect motor reactions to the same extent as the target-like singleton.

\section{Method}

\section{Participants}

Fifteen people (14 female and 1 male) took part in the experiment. Three of them were excluded from the analyses because of a high incidence of errors (> 35\%) in some RT part conditions.

\section{Stimuli and procedure}

The stimuli were the same as in Experiment 1, with the following exceptions. The intact square was defined 
as the distractor. A square outline with gaps on the top and bottom edges was defined as a target (or target-like prime), and a square outline with gaps to the left and the right was defined as an irrelevant singleton. The irrelevant singleton was never present in the target display.

The sequence was the same as in Experiment 1, except for the following changes. One of two singletons (target-like or irrelevant) appeared in one (always the same) pair of the priming sequence $100 \mathrm{~ms}$ before the target (i.e., SOA $=100 \mathrm{~ms}$ ). In compatible trials, irrelevant or target-like singletons and the target occurred on the same side. In incompatible trials, the singletons and the target were displayed on opposite sides. Each prime was displayed for $7 \mathrm{~ms}$ with an SOA of $33 \mathrm{~ms}$. Target displays were presented for $107 \mathrm{~ms} .50$ trials for each of the five conditions were presented (relevance of singleton $x$ compatibility plus one neutral condition). In neutral trials, a sequence of four pairs of intact squares preceded the main stimulus.

After this part, participants performed a prime identification (PI) task. After each sequence, participants had to report which singleton appeared in the prime display (gaps above and below, or right and left). 40 trials for each condition were presented (in total 160 trials; there was no neutral prime condition). No correctness feedback was provided ${ }^{3}$.

\section{Results}

\section{Identification}

PCIs averaged across type of singleton, separately for compatible and incompatible trials, ranged from $41 \%$ to $80 \%$ (mean $57 \%$ ) 4 . The correlation between the priming effect and PCI just failed to reach significance $(r=.55, p=.06)$. As in Experiment 1 , we included all data in our analyses. The pattern of results for participants whose prime identification was at chance level (see Appendix) did not substantially differ from the pattern of results obtained for all participants.

\section{Reaction times}

Responses were faster on compatible than on incompatible trials (317 vs. $370 \mathrm{~ms}$ ), $F(1,11)=142.6$, $p<.001$. RT for neutral trials was $342 \mathrm{~ms}$. Importantly, however, the priming effect depended on the type of singleton: RT(incompatible) - RT(compatible) $=66$ vs. $38 \mathrm{~ms}$; the interaction between compatibility and type of singleton was significant, $F(1,11)=47.4$, $p<.001$ (see Fig. 2). Separate analyses per type of singleton revealed that RTs on compatible trials were shorter than on incompatible trials, $F(1,11)=152.3$, $p<.001 ; F(1,11)=84.3, p<.001$ for relevant and irrelevant singletons, respectively. No further effects were significant.

Error rate was higher on incompatible than on compatible trials: 12 vs. $1 \%, F(1,11)=24.5$, $p<.001$. Error rate for neutral trials was $2 \%$. Mean error rate was also higher for irrelevant than for relevant singletons, 7 vs. $5 \%, F(1,11)=6.3, p<.05$. Importantly, the priming effect [PC(incompatible)$\mathrm{PC}$ (compatible)] depended on the type of singleton; Type of singleton $x$ Compatibility: $F(1,11)=18.5$, $p=.001$. An additional ANOVA performed separately for both types of singleton revealed that the priming effect was significant for both types of singleton, but it was larger when the target-like singleton was displayed in the priming sequence, 14 vs. $0 \%$, $F(1,11)=30.0, p<.001$, than when the irrelevant singleton was displayed (10 vs. $1 \%), F(1,11)=14.1$, $p<.05$, respectively.

\section{DISCUSSION}

In Experiment 2, two different types of singleton (target-like vs. irrelevant) were inserted into the priming sequence. Both types were thought to be potentially about equally effective with regard to bottom-up attentional capture, whereas only one type of them was considered to hold the potential to capture attention in a top-down manner. Both types of singleton induced a priming effect, but the effect evoked by the irrelevant singleton was significantly smaller than the effect evoked by the target-like singleton (significant Type of singleton $x$ Compatibility interaction). On the one hand, these results support the top-down view of attentional capture, as effects were largest for target-like singletons. However, as a compatibility effect was also found with the irrelevant singleton, these data additionally point to the contribution of bottom-up attentional capture. One might argue that the assumption that both types of singletons would induce a comparable capture effect is a matter of debate. Nevertheless, the data show that top-down attentional capture in itself is insufficient to account for the observed data. In other words, these data confirm that there is some contribution of bottom-up attentional capture.

One could argue that the priming effect evoked by the irrelevant singleton can be attributed to an extension of the target's definition (to squares with gaps in general). This issue will be considered in the General Discussion. 


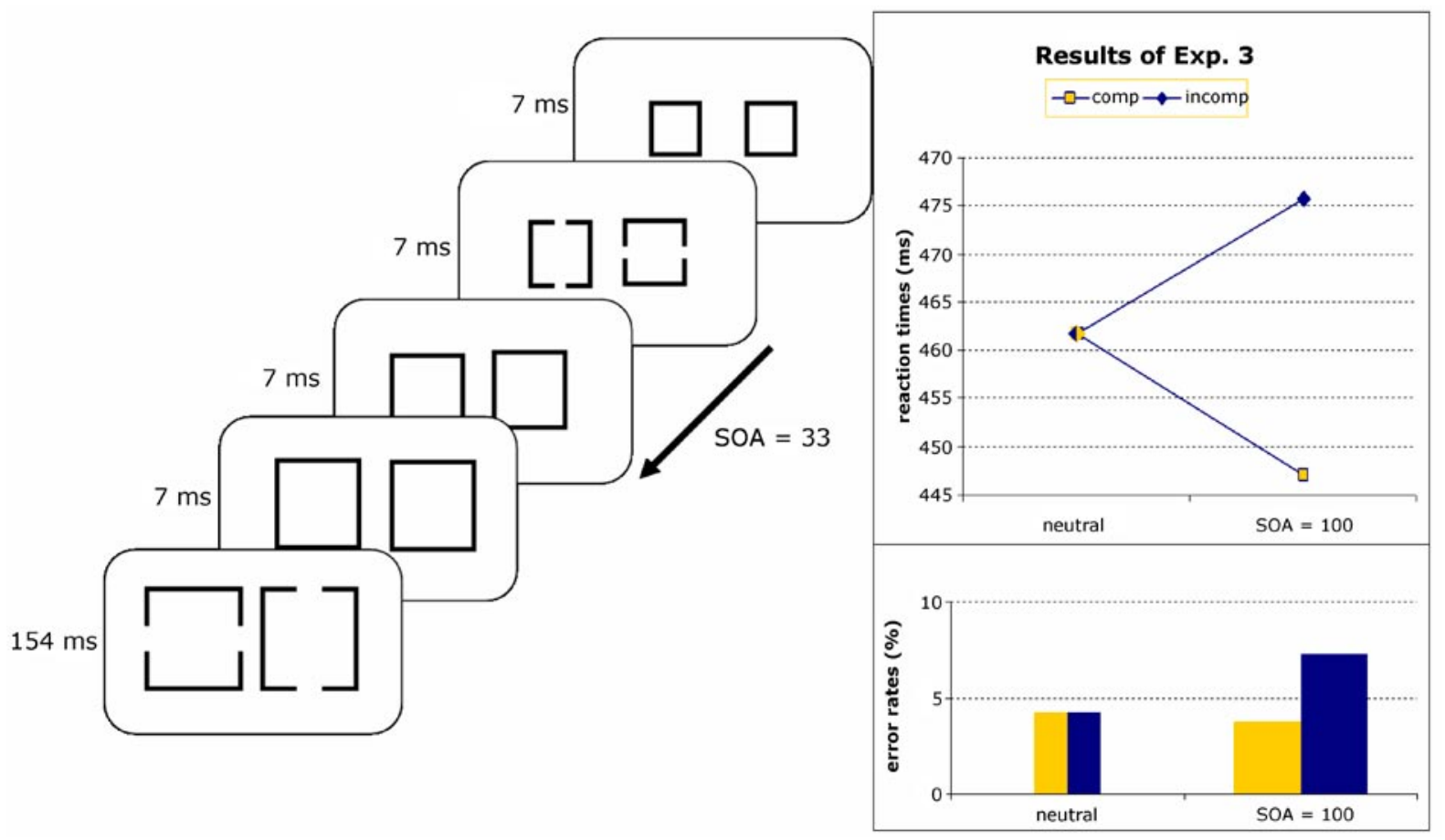

Figure 3.

Left: The sequence of stimuli used in Experiment 3, showing an example of an incompatible trial requiring a left hand reaction. Right: Reaction times and error rates in Experiment 3 as a function of compatibility.

\section{EXPERIMENT 3}

In Experiment 3, the question under investigation was whether top-down attentional capture can still be demonstrated when the role of bottom-up attentional capture is absent, or at least minimized as much as possible. Again, two different singletons were inserted into the priming sequence, one target-like singleton and one irrelevant singleton. Unlike Experiment 2, both singletons were displayed simultaneously in one pair of the priming sequence. In addition, the target display consisted of a target and a distractor that was comparable to the irrelevant singleton (i.e., it had gaps at the same location). The assignment of target type (squares with gaps at top and bottom or with gaps to the left and the right) was varied between participants.

According to the bottom-up attentional capture hypothesis, no priming effects should remain as both types of singletons are about the same in their capacity to induce bottom-up attentional capture. The top-down view of attentional capture, however, would predict the usual priming effect.

\section{Method}

\section{Participants}

Thirty-three students ( 4 male, 29 female) took part in the experiment. One participant was excluded be- cause of a very high level of errors (> 50\%) in some conditions of the RT part.

\section{Stimuli and procedure}

The stimuli were the same as in Experiment 2, but the assignment of target types was varied between participants. For approximately half of the participants, the square with gaps to the left and the right was defined as the target, whereas the square with gaps on top and bottom was defined as distractor, and for the other half of the participants, this assignment was reversed.

The procedure was the same as in Experiment 2 , except for the following changes. Both singletons appeared always in the same pair of the priming sequence $100 \mathrm{~ms}$ before the main stimulus (i.e., $\mathrm{SOA}=100 \mathrm{~ms}$ ) and both types of square occurred in the target display (Fig. 3). The target display stimulus was presented for 154 ms. Sixty trials were presented for each of the three conditions, that is, neutral, compatible, and incompatible.

In the prime identification task, participants were asked whether the target-like singleton in the prime display occurred to the left or to the right of fixation. Participants received a total of 80 trials. There were no neutral trials. No correctness feedback was provided $^{3}$. 


\section{Results}

\section{Identification}

Averaged across compatible and incompatible trials, PCIs ranged from $45 \%$ to $66 \%{ }^{5}$. For seven participants, PCIs were higher than chance. The correlation between the priming effect and PCI was significant, $r=0.4, \mathrm{p}<.05$.

\section{Reaction times}

Responses were faster on compatible trials than on incompatible trials, 447 vs. $476 \mathrm{~ms}, F(1,31)=31.4$, $p<.001$. Mean reaction time registered in the neutral condition was $462 \mathrm{~ms}$ (Fig. 3). Target type factor was insignificant, $F(1,30)<1$.

A similar pattern of results was found for error rates. Errors were committed more often on incompatible than compatible trials, 7 vs. $4 \%, F(1,31)=21.9$, $p<.001$. The error rate on neutral trials was $4 \%$.

\section{Discussion}

In Experiment 3, we investigated what happens when target-like singletons and irrelevant singletons are displayed simultaneously in a prime display. Responses were faster and more accurate on compatible trials than on incompatible trials, which accords with the top-down view of attentional capture. One could still argue, that bottom-up effects for both types of singleton are not necessarily the same. One aspect of the data from Experiment 3, however, indicates that this objection is unlikely because if it were true, then topdown attentional capture would have been affected to some extent by the assignment of target type. This was not the case.

\section{GENERAL DISCUSSION}

The present experiments were designed to determine the contributions of bottom-up and top-down attentional capture in the subliminal priming effect under conditions in which both types of attentional capture, either due to discontinuities or due to feature overlap with the target, may account for the observed results. We focused on the paradigm employed by Jaśkowski et al. (2003) where the target display, consisting of two stimuli, one to the left and one to the right of fixation, was preceded by a sequence of four displays, which were made unrecognizable by metacontrast masking.

In Experiment 1, we investigated what happens when the hypothetical effects of bottom-up and top-down attentional capture are placed in opposition. Target displays were preceded by displays consisting only of target-like replicas, and one display that consisted of an irrelevant singleton and a target-like replica. Our results were well in accordance with the conception of top-down attentional capture, as responses were faster when the target was preceded by an irrelevant singleton on the opposite side, as compared to when it occurred on the target side. Despite the clarity of this result, this observation does not rule out the possible contribution of bottom-up attentional capture, as the results only indicate that top-down attentional capture is much stronger than bottom-up attentional capture.

The possible contribution of bottom-up attentional capture was further examined in Experiment 2 by comparing the influence of target-like and irrelevant singletons in the priming sequence. The results of Experiment 2 showed that both types of singletons induced a priming effect, which confirms the view that both top-down and bottom-up attentional capture play a role. The reduced effect for the irrelevant singletons indicates that the contribution of bottom-up attentional capture is indeed smaller than the contribution of top-down attentional capture, which accords with our previous suggestion. An implication of the latter suggestion is that top-down attentional capture should still be present when the possible influence of bottomup attentional capture is minimized.

In Experiment 3, this issue was examined by presenting target-like and irrelevant singletons on opposite sides in the same display, which should reveal the remaining contribution of top-down attentional capture because both types of singleton are equal in their capacity to invoke bottom-up attentional capture. A priming effect was indeed obtained. The observed effect was smaller than the effect with target-like singletons in Experiment 2 [significant Compatibility $x$ Type of experiment interaction, $F(1,42)=18.2$, $p<.001]$, which suggests that part of the priming effect in that condition resulted from bottom-up attentional capture. Together, these three experiments show that priming effects observed in the paradigm by Jaśkowski et al. (2003) are probably due to the mutual contribution of top-down and bottom-up attentional capture, although the results additionally indicate that the contribution of top-down attentional capture is larger than the contribution of bottom-up attentional capture.

One might raise the objection that the priming effect evoked by the irrelevant singleton in Experiment 2 can be attributed to an extension of the target's definition to squares with gaps in general. This would imply confusion of the irrelevant singleton with the target figure, 
thereby providing an alternative explanation for bottom-up attentional capture. This reasoning, however, seriously distorts the original conception of top-down attentional capture implied by Folk et al. (1992), as the amount of feature overlap becomes a matter of debate. Moreover, if there was confusion about the target, severe problems should have occurred for participants in Experiment 3, which was clearly not the case. Thus, in our opinion, the priming effect with irrelevant singletons in Experiment 2 should indeed be ascribed to bottom-up attentional capture.

In the introduction to this article, we indicated that the assumed relation between the DPS hypothesis and top-down attentional capture implies that attention is captured by stimuli resembling the target, thereby matching top-down action plans for features. One might argue that this view implies that the locus of priming is at a pre-motoric level, and in this way deviates from the original proposal that free parameters of responses are specified. However, given the strong link that has been proposed between attention and the selection of action (e.g. Deubel \& Schneider, 1996), attentional selection may be considered as a kind of response selection. Obviously, this view emphasizes a role of attention in the control of actions rather than being vital for access to short term memory and consciousness, which is often implied by explanations for phenomena such as change blindness (Simons \& Levin, 1997). Moreover, the idea that topdown attentional capture would result in more conscious perception would clearly be at odds with the finding that primes are not consciously perceived (or are hardly consciously perceived). Clearly, specifying the level at which priming exerts its effect may provide important clues about the precise underlying mechanism. Based on our experiments, it may be concluded that top-down attentional capture only provides part of the answer.

With regard to the success of subliminal priming, metacontrast masking was not always as efficient as we expected. In some experiments, about half of the participants could identify or detect a masked object better than may be expected on the basis of chance. Thus, we cannot exclude the possibility that participants were aware of the primes to some extent. The high percent of correct identification or detection could be caused by strongly focused attention and perceptual learning during the PI part (especially in Experiment 1 , where feedback about correctness was provided). As mentioned earlier, there was, however, no reliable correlation between PCI level and priming effect in Experiment 1 and 2 . The lack of a correlation between
PCI and priming effect was also observed by Vorberg and coworkers (Vorberg, Mattler, Heinecke, Schmidt, \& Schwarzbach, 2004). These findings suggest that the observed priming effect is not due to awareness of the primes on a specific proportion of trials.

As mentioned in our introduction, a similar issue was investigated by Ansorge and Neumann (2005). In general, the results of our experiments corroborate their findings. In their Experiments 4 and 5, the target display contained four small squares located in the corners of an imaginary larger square. The target was defined as the square with two adjacent horizontal bars, one above and one below the square. The target display was preceded by a prime display, which also contained four (slightly smaller) squares, in such a way that each square from the prime display fitted the corresponding square in the target display. Thus, the target display masked the prime by metacontrast masking. In Experiment 4, participants had to decide whether the target was above or below fixation (the fixation point was at the center of the displays). Ansorge and Neumann found that responses were faster for compatible primes (i.e. when prime and target both occurred above or below fixation) than for incompatible primes. The bottom-up attentional capture hypothesis would predict facilitation only when the prime and target occurred at the same location. They also found facilitation, however, when both the prime and the target occurred above fixation, but at different sides of fixation, which accords with the DPS hypothesis. Nevertheless, responses were fastest when prime and targets occurred at the same location, indicating that bottom-up attentional capture plays a role.

On the basis of our findings, it may be concluded that although DPS seems to be the main mechanism underlying priming, bottom-up attentional capture contributes to this phenomenon. This conclusion is consistent with the findings of Ansorge and Neumann (2005), who, despite considerable differences in the employed methods, came up with a highly comparable conclusion.

\section{Notes}

1 Obviously, participants' intentions may play a role when they are set for a specific singleton (Bacon \& Egeth, 1994).

${ }^{2}$ An earlier examination of this issue was already reported by Ansorge and Neumann (2005): they showed that attentional capture triggered in a bottom-up manner cannot be the exclusive mechanism for subliminal priming.

${ }^{3}$ In Experiments 2 and 3, we excluded the correctness feedback provided in Experiment 1 for the fol- 
lowing reasons. By means of the correctness feedback we wanted to keep the participants' motivation at a high level. This is especially important when the prime identification part is relatively long, as in Experiment 1 (double presentation of stimuli in all 160 trials). This was no longer the case in Experiments 2 and 3. Moreover, we observed that correctness feedback remarkably improved the effectiveness of the participants' guessing through perceptual learning (see also Schubö et al., 2001; Sobieralska \& Jaśkowski, 2005). This left us with the problem of not knowing whether participants saw primes in the RT part or learned quickly during the PI part. As already mentioned, the pattern of results in all three experiments was the same regardless of whether we analyzed data of all participants or only of those whose PCIs were not significantly higher than chance level.

4 For some participants, a high level of correct responses (about $80-100 \%$ ) for target-like replica presentation and a low level for irrelevant singleton display $(0-25 \%)$ was observed. A likely reason for this is that the participants could not determine which singleton was presented in the prime display, and they favored one of the possible responses.

${ }^{5}$ For some participants, a high level of correct identifications (about $80-90 \%$ ) on compatible trials, and a low level on incompatible trials (5-25\%), was observed. A likely reason for this is that they could not determine where the masked target figure was located in the priming sequence, and therefore they chose the target location in the main stimulus (which should have been ignored).

\section{Appendix}

Using a Chi-square test, we calculated the critical PCI for a given number of trials in the prime identification parts. This value determines if a given PCI was significantly higher than chance level (50\%). These critical values were $71 \%$ in Experiment 1, and $60.9 \%$ in Experiments 2 and 3. Percentages of correct identifications/detections (PCI) were calculated for each participant separately for each condition.

\section{Selected results of Experiment 1}

Only 10 out of 18 participants in Experiment 1 detected the irrelevant singleton at chance level in each of eight conditions. In the main text, all data of the 18 participants were presented together. Here, we report reaction times and error rates of these 10 participants. As we mentioned earlier, the patterns of results for both analyses are similar.
Mean RTs were shorter for compatible than for incompatible trials, 390 vs. $454 \mathrm{~ms}, F(1,9)=128.0$, $p<.001$. RT for neutral trials was $412 \mathrm{~ms}$. RTs were independent of SOA, but the priming effect depended on SOA, being largest for medium (105 and $70 \mathrm{~ms}$ ) SOAs: RT(incompatible) - RT(compatible) $=81$ and 77 ms respectively; Compatibility $\mathrm{x}$ SOA interaction: $F(3,27)=7.2, p<.001$.

Error rates were higher on incompatible than on compatible trials (12 vs. $2 \%), F(1,9)=15.0$, $p<.05$. RT for neutral trials was $5 \%$. No other significant main effects or interactions were found.

\section{Selected results of Experiment 2}

The data of six participants did not exceed chance level in the PI task. Again, the pattern of results is similar to the analysis reported in the main text in which all participants were included.

Responses were faster on compatible than on incompatible trials ( 314 vs. $358 \mathrm{~ms}$ ), $F(1,5)=72.3$, $p<.001$. RT for neutral trials was $335 \mathrm{~ms}$. It is impor-tant to note, however, that the priming effect depended on the type of singleton: RT(incompatible) - $\mathrm{RT}$ (compatible) = 57 vs. $33 \mathrm{~ms}$; the interaction between compatibility and type of singleton was significant, $F(1,5)=14.4, p<.05$. Separate analyses for each type of singleton revealed that RTs on compatible trials were shorter than on incompatible trials, $F(1,5)=79.9, p<.001 ; F(1,5)=30.2, p<.05$ for relevant and irrelevant singletons, respectively. No further significant main effects were found.

Error rate was higher on incompatible than on compatible trials: 12 vs. $1 \%, F(1,5)=22.5, p<.05$. Error rate for neutral trials was $2 \%$. Importantly, the priming effect [PC(incompatible) - PC(compatible)] depended on the type of singleton; Type of singleton $x$ Compatibility, $F(1,5)=12.9, p<.05$. An additional ANOVA performed for both types of singleton separately revealed that the priming effect was significant for both types of singletons, but it was larger when the target-like singleton was displayed in the priming sequence, 15 vs. $0 \%, F(1,5)=29.4, p<.05$; than when the irrelevant singleton was displayed (9 vs. $1 \%$ ), $F(1,11)=9.4, p<.05$, respectively.

\section{Selected results of Experiment 3}

Twenty-five out of 32 participants of Experiment 3 identified the masked figure at a level that did not differ from chance. Here, we report reaction times and error rates of these 25 participants. As we mentioned earlier, patterns of results were similar to those reported in the main text. 
Responses were faster in compatible than in incompatible trials, 464 vs. $489 \mathrm{~ms}, F(1,24)=22.6$, $p<.001$. Mean reaction time registered in the neutral condition was $476 \mathrm{~ms}$.

A similar pattern of results was found for error rate. Errors were committed more often on incompatible than compatible trials, 8 vs. $4 \%, F(1,24)=18.1$, $p<.001$. The error rate on neutral trials was $4 \%$.

\section{References}

Ansorge, U., \& Heumann, M. (2003). Top-down contingencies in peripheral cuing: the roles of color and location. Journal of Experimental Psychology: Human Perception and Performance, 29, 937-948. Www

Ansorge, U., Heumann, M., \& Scharlau, I. (2002). Influences of visibility, intentions, and probability in a peripheral cueing task. Consciousness and Cognition, 11, 528-545. WwW

Ansorge, U., \& Neumann, O. (2005). Intentions determine the effect of metacontrast-masked primes: A test of direct parameter specification in a peripheral cueing task. Journal of Experimental Psychology: Human Performance and Perception, 31, 762-777. Www

Bacon, W. F., \& Egeth, H. E. (1994). Overriding stimulus-driven attentional capture. Perception \& Psychophysics, 55, 485-496. WwW

Deubel, H., \& Schneider, W. X. (1996). Saccade target selection and object recognition: Evidence for a common attentional mechanism. Vision Research, 36, 1827-1837. WwW

Folk, C. L., Leber, A. B., \& Egeth, H. E. (2002). Made you blink! Contingent attentional capture produces a spatial blink. Perception \& Psychophysics, 65, 741-753. $\underline{\omega w}$

Folk, C. L., Remington, R. W., \& Johnston, J. C. (1992). Involuntary covert orienting is contingent on attentional control settings. Journal of Experimental Psychology: Human Perception and Performance, 18, 1030-1044. WWw

Franconeri, S. L., Simons, D. J., \& Junge, J. A. (2004). Searching for stimulus-driven shifts of attention. Psychonomic Bulletin \& Reviews, 11, 876-881. [Ww]

Gibson, B. S., \& Jiang, Y. (1998). Surprise! An unexpected color singleton does not capture attention in visual search. Psychological Science, 9, 176-182 .

Jaśkowski, P., Skalska, B., \& Verleger, R. (2003). How the self controls its "automatic pilot" when processing subliminal information. Journal of Cognitive Neuroscience, 15, 911-920. $\underline{\mathrm{WWW}}$

Jaśkowski, P., van der Lubbe, R. H. J., Schlotterbeck, E., \& Verleger, R. (2002). Traces left on visual se- lective attention by stimuli that are not consciously identified. Psychological Science, 13, 48-54. www

Klotz, W., \& Neumann, O. (1999). Motor activation without conscious discrimination in metacontrast masking. Journal of Experimental Psychology: Human Perception and Performance, 25, 976-992.

Klotz, W., \& Wolff, P. (1995). The effect of a masked stimulus on the response to the masking stimulus. Psychological Research, 58, 92-101. Www

Kunde, W. (2003). Sequential modulations of stimulusresponse correspondence effects depend on awareness of response conflict. Psychonomic Bulletin \& Review, 10, 198-205. WWw

Leuthold, H., \& Kopp, B. (1998). Mechanisms of priming by masked stimuli: Inferences from event-related brain potentials. Psychological Science, 9, 263-269.

Lingnau, A., \& Vorberg, D. (2005). The time-course of response inhibition in masked priming. Perception \& Psychophysics, 67, 545-557. Www

Mattler, U. (2003). Priming of mental operations by masked stimuli. Perception \& Psychophysics, 65, 167-187. WWW

McCormick, P. A. (1997). Orienting attention without awareness. Journal of Experimental Psychology: Human Perception and Performance, 23, 168-180. Www

Miller, J. (1989). The control of attention by abrupt visual onsets and offsets. Perception \& Psychophysics, 45, 567-571. |WWW

Miśkiewicz, A., Skalska, B., \& Jaśkowski, P. (2002). Paradygmat wielokrotnego prymowania podprogowego dla bodźców prezentowanych centralnie i peryferyjnie [Multiple priming by means of centrally and peripherally presented subliminal stimuli]. Poster presented at conference. Automatisms - New Perspectives, 3rd Jadwisin Colloquium, 20-22 June 2002.

Neumann, O. (1990). Direct parameter specification and the concept of perception. Psychological Research, 52, 207-215. WWw

Neumann, O., Ansorge, U., \& Klotz, W. (1998). Funktionsdifferenzierung im visuellen Kortex: Grundlage für motorische Aktivierung durch nicht bewußt wahrgenommene Reize? [Functional differentiation in the visual cortex: Basis for motor activation by not consciously perceived stimuli]. Psychologische Rundschau, 49, 185-196.

Neumann, O., \& Klotz, W. (1994). Motor responses to nonreportable, masked stimuli: Where is the limit of direct parameter specification? In C. Umiltá \& M. Moscovitch (Eds.), Conscious and unconscious information processing (pp. 123-150). Cambridge, MA: 
MIT Press.

Posner, M. I., \& Cohen, Y. (1984). Components of visual orienting of attention. Journal of Neuroscience, 4, 1863-1874.

Scharlau, I., \& Ansorge, U. (2003). Direct parameter specification of an attention shift: Evidence from perceptual latency priming. Vision Research, 43, 1351-1363. WwW

Schlaghecken, F., \& Eimer, M. (2002). Motor activation with and without inhibition: Evidence for a threshold mechanism in motor control. Perception \& Psychophysics, 64, 148-162. WWW

Schubö, A., Schlaghecken, F., \& Meinecke, C. (2001). Learning to ignore the mask in texture segmentation tasks. Journal of Experimental Psychology: Human Perception and Performance, 27, 919-931. [www

Simons, D. J., \& Levin, D. T. (1997). Change blindness. Trends in Cognitive Sciences, 1, 261-267.

Skalska, B., Gierszewska, P., Miłkowska, M., Okulicz, J., \& Jaśkowski, P. (2004). Neutral primes can modify subliminal priming effect. Poster presented on European Conference on Visual Perception, 22-26 August 2004.

Sobieralska, K., \& Jaśkowski, P. (2005). How perceptual learning influence the subliminal priming ef- fect? Poster presented on European Conference on Visual Perception, 22-26 August 2005.

Theeuwes, J. (1991). Exogenous and endogenous control of attention: The effect of visual onsets and offsets. Perception \& Psychophysics, 49, 83-90. WWW

Theeuwes, J. (1992). Perceptual selectivity for color and form. Perception \& Psychophysics, 51, 599-606. WwW

Theeuwes, J. (2004). Top-down search strategies cannot override attentional capture. Psychonomic Bulletin \& Review, 11, 65-70.

van der Lubbe, R. H. J., \& Postma, A. (2005). Interruption from irrelevant auditory and visual onsets even when attention is in a focused state. Experimental Brain Research, 164, 464-471. $\underline{\underline{W W} \mid}$

Vorberg, D., Mattler, U., Heinecke, A., Schmidt, T., \& Schwarzbach, J. (2004). Invariant time course of priming with and without awareness. In C. Kaernbach, E. Schröger, \& H. Müller (Eds.), Psychophysics beyond sensation: Laws and invariants of human cognition (pp. 271-288). Mahwah, NJ, US: Lawrence Erlbaum Associates. 